\title{
DETECCIÓN DE RICKETTSIA RICKETTSII BRUMP (RICKETTSIALES: RICKETTSIACEAE) EN LA GARRAPATA CAFÉ DEL PERRO RHIPICEPHALUS SANGUINEUS LATREILLE (IXODIDA: IXODIDAE) EN LA COMARCA LAGUNERA, ZONA REEMERGENTE DE FIEBRE MANCHADA EN MÉXICO
}

\author{
DETECTION OF RICKETTSIA RICKETTSII BRUMPT (RICKETTSIALES: \\ RICKETTSIACEAE) IN THE BROWN DOG TICK RHIPICEPHALUS SANGUINEUS \\ LATREILLE (IXODIDA: IXODIDAE) IN THE COMARCA LAGUNERA, \\ A REEMERGENT SPOTTED FEVER ZONE IN MEXICO
}

\section{Antonio CASTILlo-MARTÍnEZ, SARaí M. CUETO-MEDINA, María Teresa VALDÉS-PEREZGASGA, FrANCISCO J. SÁNCHEZ-RAMOS, JAVIER LÓPEZ-HERNÁNDEZ, SERGIO HERNÁNDEZ-RODRÍGUEZ* Y ALDo I. ORTEGA-MORALES}

\author{
Universidad Autónoma Agraria Antonio Narro Unidad Laguna. Departamento de Parasitología. Periférico Raúl \\ López Sánchez s/n, col. Valle Verde, CP. 27054. Torreón, Coahuila, México <acm_sultan@hotmail.com>; \\ <sary_cueto@hotmail.com>, <cebolla_55@hotmail.com>; <fjsr1958@hotmail.com>; <marjav61@hotmail.com>, \\ <agrortega@hotmail.com>. \\ * Autor de correspondencia: <sergiohr39@hotmail.com>. \\ Recibido: 07/10/2016; aceptado 04/05/2017 \\ Editor responsable: Gabriel Villegas.
}

Castillo-Martínez, A., Cueto-Medina, S. M., Valdés-Perezgasga, M. T., Sánchez-Ramos, F. J., López-Hernández, J., Hernández-Rodríguez, S. y Ortega-Morales, A. I. (2017). Detección de Rickettsia rickettsii Brumpt (Rickettsiales: Rickettsiaceae) en la garrapata café del perro Rhipicephalus sanguineus Latreille (Ixodida: Ixodidae) en la Comarca Lagunera, zona reemergente de Fiebre Manchada en México. Acta Zoológica Mexicana (n.s.), 33(2), 339-344.

RESUMEN. La Fiebre Manchada de las Montañas Rocosas es una enfermedad reemergente en la Comarca Lagunera, ya que en los últimos años se han reportado numerosos casos en pacientes humanos. Para detectar la presencia de Rickettsia rickettsii (Brumpt, 1922) en la garrapata café del perro, se realizaron colectas aisladas en siete áreas rurales y una área periurbana de la Comarca Lagunera de Coahuila y Durango, durante junio 2015 a febrero 2016. Se colectaron de manera directa 840 garrapatas hembras a repleción sobre 168 perros domésticos (cinco garrapatas por perro), las cuales se depositaron en viales de $2 \mathrm{ml}$. Las garrapatas se llevaron al Laboratorio de Parasitología de la Universidad Autónoma Agraria Antonio Narro Unidad Laguna, donde se identificaron como Rhipicephalus sanguineus (Latreille, 1806). Para el análisis molecular se eligieron al azar 3 garrapatas por muestra para conformar 195 pools, en cada uno de los cuales se realizó la extracción de órganos internos y contenido estomacal. Para obtener ADN de cada pool se empleó la técnica del CTAB, se amplificó el gen gltA mediante ensayos de PCR usando un termociclador y un iniciador genérico (Forward: RpCS.877p, Reverse: RpCS.1258n). Ocho pools resultaron positivos a Rickettsia rickettsii con una frecuencia del 6.9\% (2/29) en la colonia Leticia Herrera (Gómez Palacio, Durango),
Castillo-Martínez, A., Cueto-Medina, S. M., Valdés-Perezgasga, M. T., Sánchez-Ramos, F.J., López-Hernández, J., Hernández-Rodríguez, S., \& Ortega-Morales, A. I. (2017). Detection of Rickettsia rickettsii Brumpt (Rickettsiales: Rickettsiaceae) in the brown dog tick Rhipicephalus sanguineus Latreille (Ixodida: Ixodidae) in the Comarca Lagunera, a reemergent Spotted Fever zone in Mexico. Acta Zoológica Mexicana (n.s.), 33(2), 339-344.

ABSTRACT. Rocky Mountain Spotted fever is a reemergent disease in the Comarca Lagunera, since numerous human cases have been reported in the last few years. In order to detect the presence of Rickettsia rickettsii Brumpt in dog's brown ticks, single collections of ticks were conducted in seven rural areas and a periurban area in the Comarca Lagunera of Coahuila and Durango from June 2015 through february 2016. Direct collection of 840 engorged female ticks on 168 home dogs was carried out (5 ticks per dog), which were contained in two $\mathrm{ml}$ vials. Ticks were identified as Rhipicephalus sanguineus Latreille in the Parasitology Laboratory of the Universidad Autonoma Agraria Antonio Narro Unidad Laguna. For the molecular analysis three ticks per sample ticks were randomly selected, forming 195 pools. Each tick was subjected to extraction of internal organs and stomachal content. CTAB technique was used on each pool in order to obtain DNA, gltA gene was amplified using PCR assays with thermocyclator and a generic primer (Forward: RpCS.844p, Reverse: RpCS. 1258n). Eight pools resulted positive to Rickettsia rickettsii with a frecuency of $6.9 \%(2 / 29)$ in the colony Leticia Herrera (Gomez Palacio, Durango), one $(1 / 26=3.85 \%)$ for Parras (Coahuila) and five pools for the municipality of Matamoros (Coahuila) in Granada (2/28=7.1\%), Alamito $(1 / 23=4.35 \%)$, Consuelo $(1 / 32=3.13 \%)$ and Vizcaya $(1 / 19=5.25 \%)$. 
un pool positivo (1/26=3.85\%) para Parras (Coahuila) y cinco pools para el municipio de Matamoros, Coahuila correspondientes a los ejidos Granada (2/28=7.1\%), Alamito $(1 / 23=4.35 \%)$, Consuelo $(1 / 32=$ $3.13 \%)$ y Vizcaya $(1 / 19=5.25 \%)$. Por medio de una secuenciación se obtuvo una identidad del 100\% a la cepa Brasileña 647 (KJ588069.1) de Rickettsia rickettsii y 99\% de similitud con las extracciones del InDRE (KU587806.1 y KT881097.1).

Palabras clave: Rickettsia rickettsii, Fiebre Manchada, garrapatas, técnica CTAB.

\section{INTRODUCCIÓN}

La garrapata café del perro Rhipicephalus sanguineus Latreille implicada en la transmisión de Fiebre Manchada fue colectada en Tlahualilo (Hunter \& Hooker, 1907), Gómez Palacio (Macías-Valadez, 1923), Pueblo Nuevo, Nazareno (Ortiz-Mariotte, 1945), Torreón (Bustamante et al., 1946; Silva-Goytia \& Elizondo, 1952) y Durango (Varela \& Aparicio, 1951); donde se encontró ectoparasitando perros y al interior de los hogares (Silva-Goytia \& Elizondo, 1952). La presencia de $R$. sanguineus en esas localidades se asoció como vector de la bacteria que ocasiona la Fiebre Manchada (Bustamante \& Varela, 1947). Los primeros casos positivos a Fiebre Manchada en humanos se registraron en el ejido Jimulco (Ortiz-Mariotte, 1945); posteriormente se presentaron otros casos en los ejidos Granada y Zaragoza, ocasionados por mordedura de garrapatas $R$. sanguineus (Bustamante et al., 1946). Durante el lapso 1975-2007, se presentaron 115 padecimientos de Fiebre Manchada (De Lara \& Cárdenas, 2008), dos padecimientos humanos sucedieron en el año

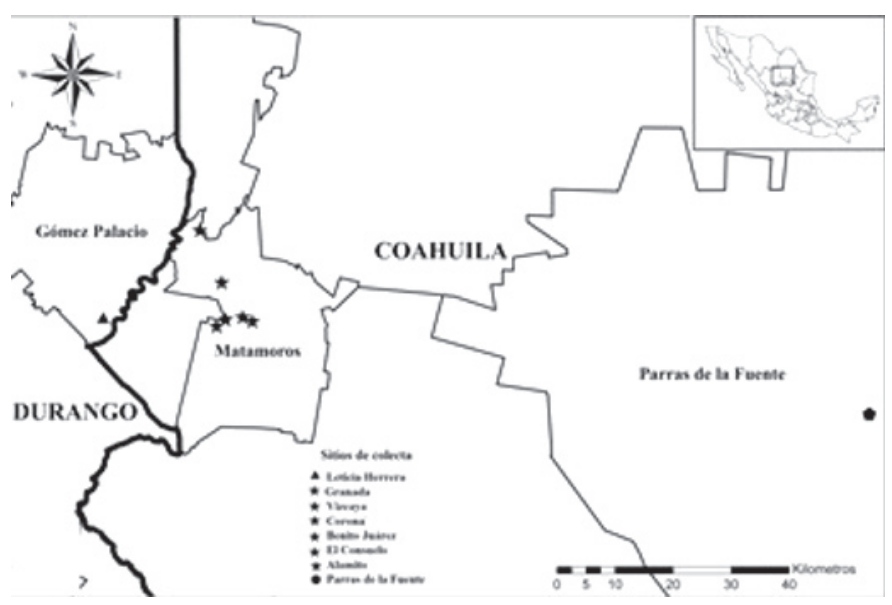

Figura 1. Áreas de la Comarca Lagunera (Coahuila y Durando) con presencia de Rhipicephalus sanguineus positivos a Rickettsia ricketsii.
By means of sequenciation 100\% identification for Rickettsia rickettsii Brazilian strain 647 (KJ588069.1) and 99\% for InDRE stractions (KU587806.1 and KT881097.1) were obtained.

Key words: Rickettsia rickettsii, spotted fever, ticks, CTAB technique.

2011 en Torreón y durante el periodo 2012-2014 se registraron 121 casos humanos de Fiebre Manchada para la Comarca Lagunera (SINAVE, 2015). Bustamante et al., (1946) aislaron dos cepas de rickettsia en garrapatas $R$. Sanguineus. Otros investigadores realizaron estudios serológicos, donde encontraron anticuerpos de $R$. rickettsii en sueros humanos, de perros y garrapatas $R$. sanguineus con similitud a la cepa Bitter Root Valley (Silva-Goytia \& Elizondo, 1953). Covarrubias et al., (2007) encontraron 10 muestras positivas a Rickettsia rickettsii mediante la técnica de Inmunofluorescencia Indirecta (IFI), en los municipios de San Pedro de las Colonias y Francisco I. Madero; mientras que Castillo-Martínez et al., (2014) registraron el hallazgo de 4 muestras positivas para Rickettsia sp. en el ejido Atalaya, obtenidas mediante PCR.

\section{MATERIALES Y MÉTODOS}

La colecta de garrapatas se realizó durante junio 2015 a febrero 2016 en seis localidades rurales del municipio de Matamoros (2531'41.77” N, 103¹3’55.38” W: 1116 msnm) y dos áreas periurbanas correspondientes a Parras de la Fuente (2526’45.09” N, 102¹0'48.82” W: 1512 msnm) y Gómez Palacio (25³4’52.46” N, 103²1’21.11” W: $1128 \mathrm{msnm}$ ), ubicadas en los estados de Coahuila y Durango como se muestra en la Figura 1 (INEGI, 2016).

Se colectaron de manera directa e intencionada para este estudio, un total de 840 garrapatas ( $(+)$ alimentadas sobre 168 perros domésticos y peridomésticos, tomando una muestra de 5 garrapatas repletas por canino (Cuadro 1), evitando el desprendimiento del gnatosoma. Las garrapatas se colocaron en microtubos de $2 \mathrm{ml}$, se depositaron en un contenedor frío para su preservación y se transportaron al Laboratorio de Biología Molecular del Departamento de Parasitología de la Universidad Autónoma Agraria Antonio Narro Unidad Laguna. Los ixódidos colectados se lavaron con agua ultrapura, posteriormente se colocaron bajo un microscopio estereoscópico para su identificación morfométrica, siguiendo las claves taxonó- 
Cuadro 1. Garrapatas (+) Rhipicephalus sanguineus colectadas en ocho localidades de la Comarca Lagunera de Coahuila y Durango, México.

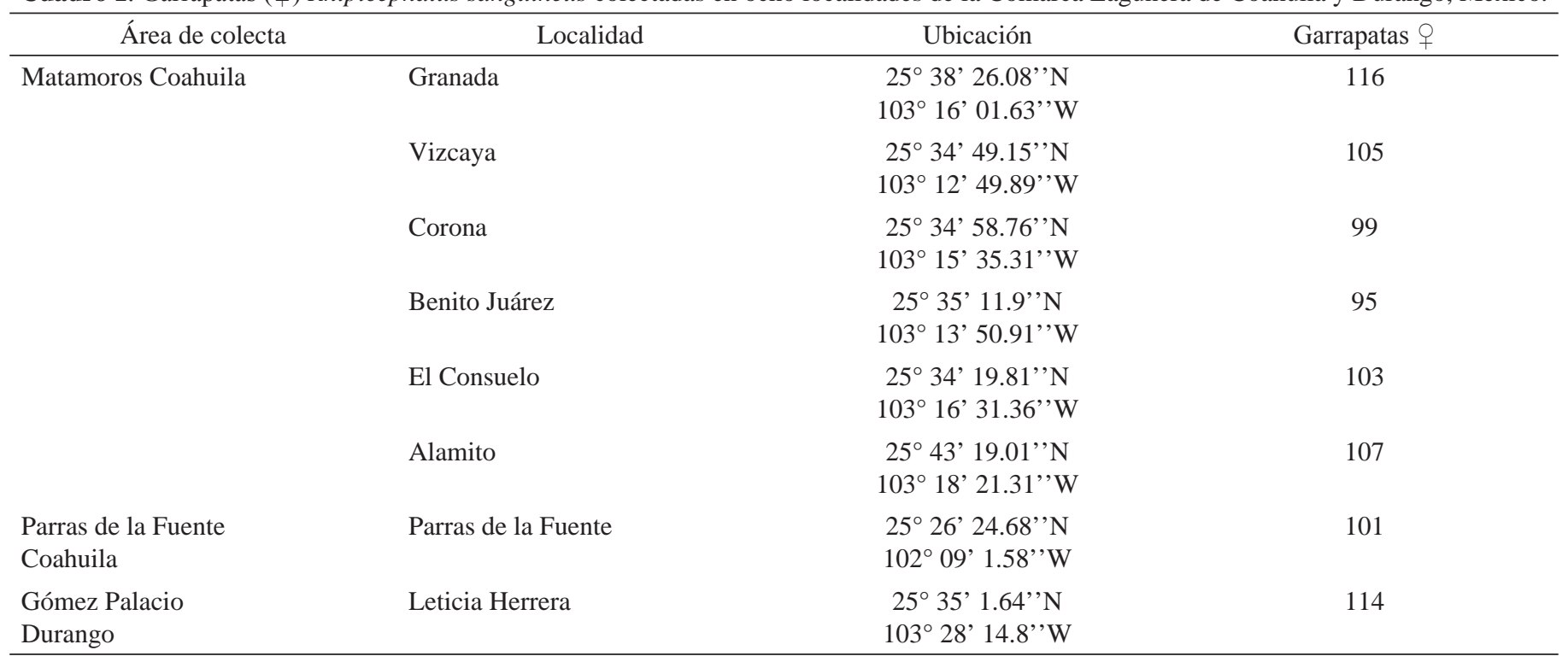

micas de Walker et al., (2014).

Para extraer el contenido interno de cada muestra se eligieron al azar tres ejemplares a repleción. Las garrapatas se diseccionaron con tijeras Iris de microdisección (BioQuip ${ }^{\circledR}$ No. 4715) previamente esterilizadas, realizando un corte en la parte posterior del abdomen; se sujetaron con unas pinzas entomológicas (BioQuip ${ }^{\circledR}$ No. 4522) desde la base de los palpos y con ayuda de una pinza curva (BioQuip ${ }^{\circledR}$ No. 4527) se deslizó presionando ligeramente la parte dorsal y ventral de los especímenes hasta la parte posterior del idiosoma. El contenido interno de cada muestra se depositó en un microtubo de $1.5 \mathrm{ml}$ con un medio líquido de agua milli-Q (Life Invitrogen ${ }^{\circledR}$, USA) y se maceraron usando pistilos de maceración de tejido (Thomas Scientific ${ }^{\circledR}$ No. TS0913X70). Los productos macerados (195 pools) se agruparon a razón de 1 muestra $=3$ ㅇ adultas a repleción (Cuadro 2).

Extracción de ADN. La extracción de ADN se realizó mediante el protocolo Cetyl Trimethyl Ammonium Bromide o CTAB (Doyle \& Doyle, 1987) con las siguientes modificaciones: a cada muestra macerada se agregaron $200 \mu$ de CTAB $2 X$, se mezclaron durante 15 seg en el vortex (Select vortexer, Select Bioproducts ${ }^{\circledR}$, U.S.A.), en seguida se colocaron los microtubos en el termoblock (Digital block heater, Select Bioproducts ${ }^{\circledR}$ U.S.A.) a 65 ${ }^{\circ} \mathrm{C}$ durante $30 \mathrm{~min}$; posteriormente se adicionaron $200 \mu \mathrm{l}$ de cloroformo frío (C4425-12, Jalmek ${ }^{\circledR}$ ) y se mezclaron por 10 seg en el vortex. Las muestras se colocaron en una centrífuga (Force micro 1624, Select Bioproducts ${ }^{\circledR}$ U.S.A.) durante $20 \mathrm{~min}$ a $13000 \mathrm{rpm}$, se transfirieron 120 $\mu \mathrm{l}$ de la fase acuosa a microtubos estériles de $1.5 \mathrm{ml}$, a las cuales se les adicionaron $200 \mu \mathrm{l}$ de isopropanol frío (P6925-13, Jalmek®) y se dejaron reposar durante $30 \mathrm{~min}$ a $-30{ }^{\circ} \mathrm{C}$ para precipitar los ácidos nucleicos. Las muestras nuevamente se centrifugaron durante $20 \mathrm{~min}$ a 13000 rpm, se decantó el isopropanol para agregar $200 \mu$ l de etanol frío (E5325-15, Jalmek®) al 70\%, se realizó otra centrifugación por 10 min a 13000 rpm; se decantó el etanol dejando secar la pastilla a temperatura ambiente durante 40 min y finalmente se resuspendió el ADN en $30 \mu \mathrm{l}$ de agua milli-Q (Life Invitrogen ${ }^{\circledR}$, USA).

Pruebas Moleculares. La calidad y cantidad de ADN obtenido por medio de nanodrop (Thermo Scientific ${ }^{\circledR}$ NanoDrop 2000) fue evaluada, resuspendiéndola en $20 \mu \mathrm{l}$ de buffer TE $1 X$ [ $400 \mu$ l EDTA (Life Invitrogen ${ }^{\circledR}$, USA) $+4 \mathrm{ml}$ Tris Base (Bio Basic Canada Inc $\left.{ }^{\circledR}\right]$ para almacenarla a $-20{ }^{\circ} \mathrm{C}$. Para las pruebas de PCR se utilizó el iniciador genérico que amplifica el gen gltA en un rango de 380-397 pb (Wood et al., 1987) para Ricketssia spp: Forward: RpCS.877p GGGGGCCTGCTCACGGCGG, Reverse: RpCS.1258n ATTGCAAAAAGTACAGTGAACA (Regnery et al., 1991). La reacción de PCR se realizó con la siguiente mezcla de reacción: $\mathrm{MgCl}_{2}$ a una concentración de $1.5 \mathrm{mM}$, buffer 10X (200 mM Tris-HCl pH 8.4 Bio Basic Canada Inc ${ }^{\circledR}$ ), dNTP’s (Life Invitrogen ®, USA) a una concentración de $0.2 \mathrm{mM}, 1.25 \mathrm{U}$ de Taq DNA polimerasa (Life Invitrogen ${ }^{\circledR}$, USA), ADN templado concentrado a $20-25 \mathrm{ng} / \mu \mathrm{l}, 20 \mathrm{pmol} / \mu \mathrm{l}$ de cada uno de los iniciadores (RpCS.877p, RpCS.1258n) y agua milli-Q (Life Invitrogen ${ }^{\circledR}$, USA) para completar un Rx de $25 \mu$ l. 
Cuadro 2. Muestras de garrapatas Rhipicephalus sanguineus, pools procesados y resultados de los análisis por PCR para la detección de Rickettsia rickettsii.

\begin{tabular}{lcccc}
\hline \multicolumn{1}{c}{ Localidad } & No. de Garrapatas & No. de pools (3qc/u) & Pools positivos & Pools negativos \\
\hline Granada & 84 & 28 & $2(7.14 \%)$ & $26(92.86 \%)$ \\
Vizcaya & 57 & 19 & $1(5.26 \%)$ & $18(94.74 \%)$ \\
Corona & 63 & 21 & 0 & $21(100 \%)$ \\
Benito Juárez & 51 & 17 & 0 & $17(100 \%)$ \\
El Consuelo & 96 & 32 & $1(3.13 \%)$ & $31(96.87 \%)$ \\
Alamito & 69 & 23 & $1(4.35 \%)$ & $22(95.65 \%)$ \\
Parras & 78 & 26 & $1(3.85 \%)$ & $25(96.15 \%)$ \\
Leticia Herrera & 87 & 29 & $2(6.90 \%)$ & $27(93.10 \%)$ \\
\hline
\end{tabular}

Las condiciones del termociclador Select Cycler II (Select BioProducts ${ }^{\circledR}$, USA) para amplificar el gen gltA fueron las siguientes: un ciclo a $95{ }^{\circ} \mathrm{C}$ durante un minuto, 35 ciclos conformados por tres procesos: desnaturalización a $95{ }^{\circ} \mathrm{C}$ (20 segundos), alineación de iniciadores a $48{ }^{\circ} \mathrm{C}$ por 30 segundos, una extensión a $60{ }^{\circ} \mathrm{C}(2 \mathrm{minu}-$ tos) y otra extensión final a $72{ }^{\circ} \mathrm{C}$ (1 min). Como control positivo se utilizó una muestra de Rickettsia rickettsii proporcionada por el Instituto de Investigaciones en Ciencias Veterinarias (UABC) y una mezcla sin ADN para el control negativo. El marcador de peso molecular (Hyperladder 100-pb - Bioline ${ }^{\circledR}$, USA), el control positivo y los productos obtenidos de PCR fueron teñidos con Gel Red (Biotium TM, USA) y observados en gel de agarosa al 1\%; para visualizar los resultados de los productos se empleó un fotodocumentador MiniBis Pro (Bio-Imaging Systems $\left.{ }^{\circledR}\right)$.

\section{RESULTADOS}

Todos los especímenes fueron consistentes con la descripción de $R$. sanguineus (Walker et al., 2014) y de estas garrapatas se obtuvieron 8 pools positivos $(8 / 195=4.1 \%)$ a Rickettsia sp. La presencia de Rickettsia sp. en los pools $(\mathrm{n}=195)$ compuesto por órganos y contenido estomacal macerado de las garrapatas a repleción, fue determinada mediante PCR empleando los iniciadores RpCS.877p y RpCS.1258n, los cuales amplificaron satisfactoriamente el gen de la citrato sintasa (gltA) en un rango de 380-397 pb (Fig. 2). La purificación y la secuenciación de los productos positivos fueron realizados por Macrogen (USA) en dos sentidos (Forward, Reverse). Los productos secuenciados mostraron una conformación de 364 pb, que al analizarse arrojaron una identidad del $100 \%$ a Rickett- sia rickettsii con la cepa Brasileña 647 (No. de acceso KJ588069.1) y 99\% de similitud a las cepas del InDRE (KU587806.1 y KT881097.1) depositados en la base de datos del GenBank.

De los pools analizados, se encontró a la bacteria Rickettsia rickettsii manifestando frecuencias altas del 7.1\% (2/28) en el ejido Granada (Matamoros, Coahuila) y 6.9\% (2/29) en la colonia Leticia Herrera (Gómez Palacio, Durango). Las otras cuatro áreas muestreadas obtuvieron menor frecuencia, donde destacan tres localidades del municipio de Matamoros, Coahuila: ejido Vizcaya $(1 / 19=5.25 \%)$, Alamito $(1 / 23=4.35 \%)$ y ejido Consuelo $(1 / 32=3.13 \%)$; así como un pool positivo $(1 / 26=3.85 \%)$ para el municipio de Parras, Coahuila (Cuadro 2).

\section{DISCUSIÓN}

El medio rural y semiurbano presenta desplazamientos de humanos, perros y otros animales domésticos del campo

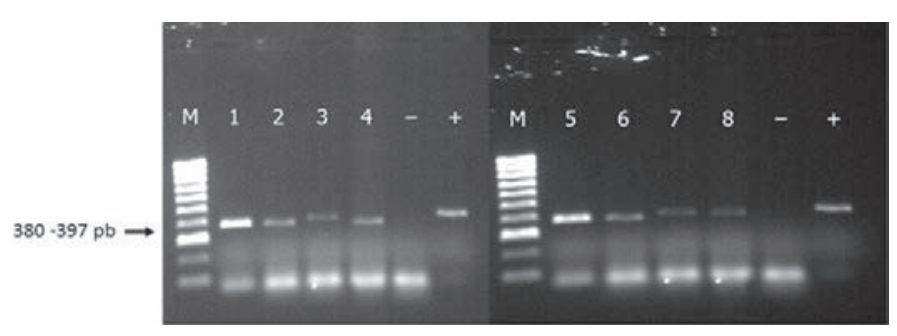

Figura 2. Productos de PCR con el gen gltA de Rickettsia sp., obtenido del ADN de pools de garrapatas $R$. sanguineus. Carriles: 1-2= Granada, 3= Vizcaya, 4= Consuelo, 5= Alamito, 6= Parras de la Fuente, 7-8= Leticia Herrera. M= marcador molecular 100-1000 pb., $+=$ Control positivo, $-=$ Control negativo sin ADN. Fragmento observado de 380-397 pb. 
al poblado; favoreciendo el transporte de garrapatas del área silvestre al hogar, donde conviven en aglomeración con otros animales domésticos, tal como lo manifiesta Bustamante (1956, 1964). Las características anteriormente descritas propician la reemergencia de rickettsiosis, cuando las condiciones ambientales de humedad y temperatura son favorables para que el vector aumente sus poblaciones y el patógeno se manifieste.

En las áreas rurales que comprenden los municipios de Matamoros (Coahuila) y Gómez Palacio (Durango), México; se encuentran cohabitando las garrapatas $R$. sanguineus, Otobius megnini (Dugès, 1884) y Argas persicus (Oken, 1818), donde la garrapata café del perro ( $R$. sanguineus) es la especie más abundante y frecuente en perros domésticos y de vida libre (Hoffmann, 1962; Castillo-Martínez et al., 2015; 2016). Existen otras especies de ixódidos (Dermacentor sp., Amblyoma sp.) que pueden estar implicadas en la transmisión de $R$. rickettsii (Guzmán-Cornejo et al., 2011; Guzmán-Cornejo et al., 2016), sin embargo, hasta el momento solo $R$. sanguineus se ha visto involucrada en el ciclo de transmisión rickettsial en la Comarca Lagunera (Bustamante \& Varela, 1947; Labruna et al., 2011); donde se han presentado múltiples casos de Fiebre Manchada en humanos (SINAVE, 2015). Los resultados de este estudio confirman la presencia de Rickettsia rickettsii como causante de Fiebre Manchada, en donde se señala a la garrapata $R$. sanguineus como vector de la bacteria $R$. rickettsii, por ser la especie de garrapata más ampliamente distribuida en la región. Lo anterior concuerda en parte con las investigaciones realizadas por Bustamante et al., (1946), quienes mediante serología aislaron la cepa Bitter Root Valley de rickettsia en garrapatas $R$. sanguineus colectadas en el Ejido Granada. Sin embargo, en esta investigación los resultados arrojaron una similitud del 100\% a la cepa Brasileña 647 (No. de acceso KJ588069.1) y 99\% de similitud a las cepas del InDRE (1098: KU587806.1 y 1075: KT881097.1), puesto que éstas últimas presentan una mutación en la posición 338 producto de la transición de una Citosina (GAGCAGG-C-CC) por una Timina (GAGCAGG-T-CC) observada en las cepas $(1098,1075)$ del InDRE.

Las modificaciones realizadas al método de obtención del pool y a la extracción de ADN, redujeron considerablemente la cantidad de sustancias inhibidoras de la PCR. La extracción y maceración de los órganos internos mostraron los mismos resultados que la trituración completa de ejemplares, pero evitando la presencia de material quitinoso en las muestras, lo cual interfiere en la centrifugación y precipitación de sólidos. Las modificaciones al protocolo del CTAB sustituyeron algunos procedimientos para acortar la metodología, se modificaron los tiempos de centrifugado y las temperaturas para la precipitación del ADN; obteniendo los mismos resultados que la técnica original de Doyle \& Doyle (1987). Al someter el ADN extraído bajo ensayos de PCR, se obtuvieron ocho muestras positivas al género Rickettsia sp. utilizando los iniciadores genéricos diseñados por Regnery et al., (1991). Los resultados positivos secuenciados (8/195=4.1\%) concuerdan con las investigaciones realizadas por Demma et al., (2005), quienes mencionan que solo del 1 al 5\% de las garrapatas están infectados por rickettsias en zonas de baja incidencia a Fiebre Manchada; sin embargo, las frecuencias son relativamente altas en cada localidad con presencia de $R$. rickettsi de acuerdo a los pools analizados.

\section{CONCLUSIÓN}

La bacteria $R$. rickettsii está presente en áreas rurales y periurbanas de la Comarca Lagunera de Coahuila y Durango y su principal vector hasta el momento es la garrapata café del perro $R$. sanguineus. El comportamiento de los casos humanos positivos a Fiebre Manchada, indican que la bacteria $R$. rickettsii se encuentra en los órganos internos de la garrapata y se comporta como un patógeno reemergente. Los movimientos del medio rural al urbano y las actividades que involucran el pastoreo de ganado, propician la diseminación de garrapatas y patógenos que causan enfermedades de interés en salud pública y veterinaria; por lo tanto se recomienda realizar más investigaciones para conocer la diversidad de ixódidos y patógenos presentes en los estados de Coahuila y Durango.

\section{LITERATURA CITADA}

Bustamante, M. (1956). Aspectos epidemiológicos de las Rickettsiasis en México: 1934 a 1954. Gaceta Médica de México, 3, 207216.

Bustamante, M. E., Varela, G. \& Ortiz-Mariotte, C. (1946). Estudios de Fiebre Manchada en México. Fiebre Manchada en la Laguna. Revista Instituto de Salubridad y Enfermedades Tropicales, 7, 39- 48.

Bustamante, M. E. (1964). Symposium sobre histoplasmosis pulmonar primaria II: Epidemiología. Gaceta Médica de México, 5, 509-518.

Bustamante, M. E. \& Varela, G. (1947). Estudios de Fiebre Manchada en México: papel del Rhipicephalus sanguineus en la transmisión de la Fiebre Manchada en la República Mexicana. Revista del Instituto de Salubridad y Enfermedades Tropicales, 8, 139-41. 
Castillo-Martínez, A., Cueto-Medina, S., Méndez-López, R., Pérez-Muñoz, R., Hernández-Rodríguez, S. \& Ortega-Morales, A. (2016). Garrapatas (Acari: Ixodidae, Argasidae) de la Comarca Lagunera en Durango, México. Entomología Mexicana, 3, 26-32.

Castillo-Martínez, A., Cueto-Medina, S. M., Hernández-Rodríguez, S., Valdés-Perezgasga, M. T., Ortega-Morales, A. I. (2015). Garrapatas peridomésticas (Acari: Ixodidae, Argasidae) de Matamoros, Coahuila, México. Entomología Mexicana, 2, 4751.

Castillo-Martínez, A., Cueto-Medina, S. M., Hernández-Rodríguez, S., Gallegos-Robles, M. A., Valdés-Perezgasga, M. T., Sánchez-Ramos, F. J. \& Ortega-Morales, A. I. (2015). Detección de Rickettsia sp. en la garrapata café del perro Rhipicephalus sanguineus (Acari: Ixodidae) en Matamoros, Coahuila, México. Acta Zoológica Mexicana (n.s.), 31, 80-83.

Covarrubias, C. J., Zavala, V. J. \& Vásquez A. J. (2007). Frecuencia de anticuerpos rickettsiales de Fiebre Manchada en pacientes febriles de los municipios San Pedro de las Colonias y Francisco I. Madero, Coahuila, México. Revista Chapingo Serie Zonas Áridas, 6, 9-16.

De Lara, H. J. \& Cárdenas, B. R. (2008). Fiebre Manchada de las Montañas Rocosas en pediatría. Revisión clínica de una serie de 115 casos. Revista de enfermedades infecciosas en pediatría, 22, 4-9.

Demma, L. J., M. S. Traeger, W. L. Nicholson, C. D. Paddock, D. M. Blau, M. E. Eremeeva, G. A. Dasch, M. L. Levin, J. Singleton, S. R. Zaki, J. E. Cheek, D. L. Swerdlow \& J. H. McQuiston. (2005). Rocky Mountain spotted fever from an unexpected tick vector in Arizona. New England Journal of Medicine, 353, 587-594.

Doyle, J. J. \& Doyle, J. L. (1987). A rapid DNA isolation procedure for small quantities of fresh leaf tissue. Phytochemical Bulletin, 19, 11-15.

Guzmán-Cornejo C., Robbins, R. G., Guglielmone, A. A., Montiel-Parra, G. \& Pérez, T. M. (2011). The Amblyomma (Acari: Ixodida: Ixodidae) of Mexico: identification keys, distribution and hosts. Zootaxa, 2998, 16-38.

Guzmán-Cornejo, C., R.G. Robbins, A.A. Guglielmone, G. Montiel-Parra, G. Rivas \& Pérez T.M. (2016). The Dermacentor (Acari, Ixodida, Ixodidae) of Mexico: hosts, geographical distribution and new records. Zookeys, 24(569), 1-22.

Hoffman, A. (1962). Monografía de los Ixodoidea de México: parte I. Revista de la Sociedad Mexicana de Historia Natural, 23, 191 pp.

Hunter, W. D. \& Hooker W A. (1907). Information concerning the North American Fever Tick, with notes on other species. U.S. Dept. Agric. Bureau of Entomology Bulletin. No. 72: 87 pp.
Instituto Nacional de Estadística y Geografía (INEGI). (2016). Mapa Digital de México. [En línea] http://gaia.inegi.org.mx/mdm6/ ?v=bGF0OjI1LjU0ODk5LGxvbjotMTAzLjQwMTkyLHo6OCxs OmMxMTFzZXJ2 aWNpb3N8dGMxMTFzZXJ2aWNpb3M= [Fecha de consulta 13/Junio/2016].

Labruna, M. M. Ogrzewalska, J. Soares, T. Martins, H. Soares, J. Moraes-Filho, F. Nieri-Bastos, A. Almeida, \& Pinter, A. (2011). Experimental Infection of Ticks with R. rickettsii. Emerging Infectious Diseases, 17, 829-834.

Macías-Valadez, S. (1923). Ensayo de una monografía sobre Ixodidos mexicanos vulgo garrapatas. Memorias de la Sociedad Científica Antonio Alzate, 41, 197-216.

National Center for Biotechnology Information (NCBI). (2016). BLAST: Basic Local Alignment Search Tool. [En línea] http:// blast.ncbi.nlm.nih.gov/Blast.cgi?PROGRAM=blastn\&PAGE_ TYPE =BlastSearch\&LINK_LOC=blasthome [Fecha de consulta 28/Mayo/2016].

Ortiz-Mariotte, C. (1945). Estudio de la Fiebre Manchada en la zona de Jimulco. Boletín Epidemiológico, 8, 26-28.

Regnery, R. L., Spruill, C. L. \& Plikatys, B. D. (1991). Genotypic identification of Rickettsiae and estimation of intraespecies sequence divergence for portions of two rickettsial genes. Journal Bacteriology, 173, 1576-1589.

Silva-Goytia, R. \& Elizondo A. (1952). Estudios sobre Fiebre Manchada en México. II. Parásitos hematófagos encontrados naturalmente infectados. Revista Mexicana de Medicina, 32(654), 278-282.

Silva-Goytia, R. \& Elizondo A. (1953). Estudios sobre Fiebre Manchada en México. III. Estudios por medio de fijación de complemento de sueros de ciertos animales domésticos de la Comarca Lagunera. Revista Mexicana de Medicina, 33(670), 76-83.

Sistema Nacional de Vigilancia Epidemiológica (SINAVE). (2015). Anuarios de Morbilidad: Fiebre Manchada. [En línea] http://www. epidemiologia.salud.gob.mx/anuario/2015/morbilidad/ enferme$\mathrm{dad} /$ distribucion_casos_nuevos_enfermedad_fuente_notificacion. [Fecha de consulta 19/ julio/ 2016].

Varela, G. \& Aparicio, A. (1951). Intestinal bacteria found in Triatoma and Ornithodoros. The American Journal of Tropical Medicine, 31, 381-382.

Walker, A. R., Bouattour, A., Camicas, J. L., Estrada-Peña, A., Horak, I. G., Latif, A. A., Pegram, R. G. \& Preston., P. M. (2014). Ticks of Domestic Animals in Africa: a Guide to Identification of Species. Bioscience Reports, Edinburgh Scotland, U.K. 221 pp.

Wood, D. O., L. R. Williamson, H. H. Winkler \& Krause, D. C. (1987). Nucleotide sequence of the Rickettsia prowazekii citrate synthase gene. Journal of Bacteriology, 169, 3564-3572. 